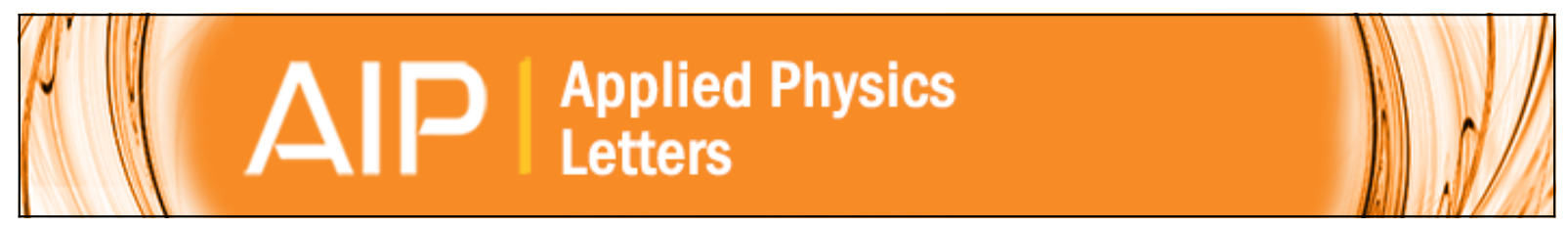

\title{
Enhanced magnetoresistance effect in layered systems
}

M. S. Ferreira, J. d'Albuquerque e Castro, R. B. Muniz, and Murielle Villeret

Citation: Applied Physics Letters 75, 2307 (1999); doi: 10.1063/1.124999

View online: http://dx.doi.org/10.1063/1.124999

View Table of Contents: http://scitation.aip.org/content/aip/journal/apl/75/15?ver=pdfcov

Published by the AIP Publishing

\section{AlP Re-register for Table of Content Alerts}

\section{Create a profile. \\ Sign up today!}




\title{
Enhanced magnetoresistance effect in layered systems
}

\author{
M. S. Ferreira, J. d'Albuquerque e Castro, and R. B. Muniz \\ Departamento de Física, Universidade Federal Fluminense, Niterói 24210-340, Brazil \\ Murielle Villeret \\ Department of Mathematics, City University, London ECIV OHB, United Kingdom
}

(Received 14 June 1999; accepted for publication 15 August 1999)

\begin{abstract}
Magnetoresistance ratios several orders of magnitude higher than those of conventional multilayers may be obtained with much smaller saturation fields in magnetic layers separated by a periodically modulated structure. Conditions for the occurrence of such effect, as well as the possible use of these systems as spin-filter devices and magnetic logical gates, are discussed. (C) 1999 American Institute of Physics. [S0003-6951(99)03941-8]
\end{abstract}

The so-called giant magnetoresistance (GMR) effect was first observed in metallic structures of alternate layers of ferromagnetic and nonmagnetic materials. ${ }^{1}$ It consists of a striking drop in the electric resistance of the system when an externally applied magnetic field changes the relative orientation of the magnetizations of the magnetic layers from antiparallel to parallel. The effect was also observed in granular materials consisting of clusters of a ferromagnetic metal embedded in a metallic matrix. ${ }^{2}$ In this case the reduction in resistance occurs when the cluster magnetizations are aligned by a magnetic field. The GMR effect is of great interest in magnetics-based technology because of its possible use in the production of devices such as ultrasensitive magnetic field sensors and read heads. For this purpose, one needs high magnetoresistance ratios and relatively low saturation magnetic fields.

In multilayer systems, the GMR effect is observed as a response to currents flowing either in the planes or perpendicularly to the planes (CPP) of the layers. It is intimately related to spin-dependent scattering which occurs both at the interfaces and within the bulk of the layers. The effect is usually larger in the CPP geometry, where the current probes all layers as it crosses the system. In addition, if sample dimensions are much smaller than the carrier mean free path, interface scattering dominates and transport in the CPP geometry occurs in the so-called ballistic regime. In the discussion of magnetoresistance (MR) it is convenient to take the spin quantization axis along that of the layer magnetizations in both ferromagnetic (FM) and antiferromagnetic (AF) configurations of the system. Then, the electric current splits into up- and down-spin components which, in the absence of spin mixing, should be added in parallel. Because of the exchange splitting in the FM layers, the potentials seen by up- and down-spin electrons as they move across the structure are different, leading to different values $\Gamma^{\uparrow}$ and $\Gamma^{\downarrow}$ of the electric conductances. $\Gamma^{\uparrow}$ and $\Gamma^{\downarrow}$, as well as the total conductance $\Gamma=\Gamma^{\uparrow}+\Gamma^{\downarrow}$, depend on the magnetic configuration of the system, and the MR ratio is usually defined as $\left(\Gamma_{\mathrm{FM}}\right.$ $\left.-\Gamma_{\mathrm{AF}}\right) / \Gamma_{\mathrm{AF}}$.

In conventional structures, in which the nonmagnetic spacer layer consists of a single metal, the MR ratio may reach values up to $220 \%^{3}$ at low temperatures. In the CPP geometry and in the ballistic regime, this ratio is basically controlled by the difference between the potentials probed by the carriers in the magnetic layers and in the spacer. Here we establish conditions under which MR ratios several orders of magnitude larger than those of ordinary multilayers can be achieved in structures with periodically modulated spacers. As discussed below, such systems may exhibit highly anisotropic electric behavior, and undergo a metal-insulator transition in the modulation direction driven by an externally applied magnetic field. The insulator behavior results from quantum reflections and interference effects within the structure, and gives rise to a phenomenal magnetoresistance (PMR) effect, which can be larger than the so-called colossal magnetoresistance $(\mathrm{CMR})^{4}$ and comparable to the enhanced GMR due to layer thickness fluctuations. ${ }^{5}$ In addition, the saturating magnetic field necessary to realize the PMR in these structures can be very weak. The combination of PMR and low saturation fields makes these systems ideal candidates for magnetic reading devices. Moreover, we show that in the FM configuration these systems behave as spin filters, which are important for technological applications. The remarkable occurrence of a magnetic-field-driven metalinsulator transition in these structures opens up the possibility of using them to produce magnetic logical gates, which are also of technological interest.

As an illustration of the behavior described above, we consider a simple system in which the magnetic and nonmagnetic materials are described by free-electron models. We examine two layers of a magnetic material separated by a periodically modulated spacer having a superlattice structure with $n$ repeats of a double layer, labeled $A$ and $B$. Thus, the spacer thickness is $L=n(a+b)$, where $a$ and $b$ are the thicknesses of the $A$ and $B$ layers. We assume CPP geometry and that transport occurs in the ballistic regime. The carrier motion then separates into components parallel and perpendicular to the layers, with energies $E_{\|}$and $E_{\perp}$, respectively. In the parallel direction, the carriers move freely and $E_{\|}$ $=\hbar^{2} k_{\|}^{2} / 2 m$, where $k_{\|}$is the modulus of the parallel wave vector. In the perpendicular direction they experience a Kronig-Penney-like potential equal to $V_{A}$ and $V_{B}$, in the $A$ and $B$ layers, respectively, and either $V_{\uparrow}$ or $V_{\downarrow}$, in the ferromagnetic layers. We emphasize that the potential profiles seen by carriers with different spin orientations depend on the magnetic configuration of the system. 


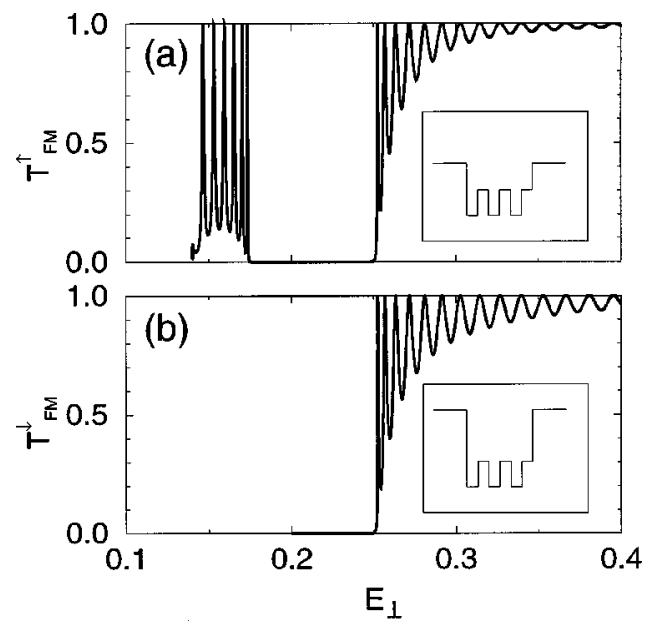

FIG. 1. Transmission coefficients $T_{\mathrm{FM}}^{\uparrow}$ (a) and $T_{\mathrm{FM}}^{\downarrow}$ (b) as functions of $E_{\perp}$. Insets show schematic representations of the corresponding potential profile. Potential parameters are already given.

Within the Landauer-Büttiker formalism, the zerotemperature conductance is given by

$$
\Gamma_{\mathrm{FM}(\mathrm{AF})}^{\sigma}=\left(e^{2} / h\right) \sum_{\mathbf{k}_{\|}} T_{\mathrm{FM}(\mathrm{AF})}^{\sigma}\left(\mathbf{k}_{\|}, E_{F}\right),
$$

where $T_{\mathrm{FM}(\mathrm{AF})}^{\sigma}\left(\mathbf{k}_{\|}, E_{F}\right)$ is the transmission coefficient in the FM (AF) configuration for carriers with spin $\sigma$, and $E_{F}$ is the Fermi energy. We remark that the contribution to the integral from each $\mathbf{k}_{\|}$corresponds to the solution of an effective onedimensional problem. In the present case, since $T_{\mathrm{FM}(\mathrm{AF})}^{\sigma}\left(\mathbf{k}_{\|}, E_{F}\right)=T_{\mathrm{FM}(\mathrm{AF})}^{\sigma}\left[E_{F}-E_{\|}\left(\mathbf{k}_{\|}\right)\right]$, the sum over $\mathbf{k}_{\|}$in the expression for each conductance can be replaced by an integral over $E_{\perp}=E_{F}-E_{\|}$up to $E_{F}$. We notice that transmission coefficients across superlattices observed as functions of the incident particle energy show gaps which result from quantum interferences within the superlattice structure. ${ }^{6}$ The number, widths, and energy positions of such gaps vary with the superlattice unit cell composition and thicknesses of its constituent layers. The presence of ferromagnets sandwiching a finite nonmagnetic superlattice imposes spindependent boundary conditions which affect the transmission coefficients across the structure. We show that it is possible to take advantage of the gaps due to the superlattice structure and of the ferromagnets to prevent carriers in one spin state from being transmitted across the system. To illustrate this point we look at the transmission coefficients for a particular example. Figures 1(a) and 1(b) show $T_{\mathrm{FM}}^{\uparrow}\left(T_{\mathrm{FM}}^{\downarrow}\right)$ as functions of $E_{\perp}$ for $n=30, V_{\uparrow}=0.14, V_{\downarrow}=0.2, V_{A}=0, V_{B}=0.12$, and $a=b=4$. Here energies and lengths are measured in atomic units. The corresponding potential profiles are schematically represented in the insets. The most interesting feature of the curves for the transmission coefficients is the presence of a transmission gap for energies above the wells/barriers in the two cases. We point out that if $V_{B}$ is set equal to $V_{A}$, as in the case of a homogeneous spacer, the gap disappears and the textbook transmission curves of quantum wells are obtained. In the curve for $T_{\mathrm{FM}}^{\uparrow}$ the gap is preceded by a transmission band, which is suppressed in the case of down-spin carriers due to the potential height in the ferromagnetic layers. As demonstrated below, such suppression plays an important role in the PMR effect. We remark that in the $\mathrm{AF}$

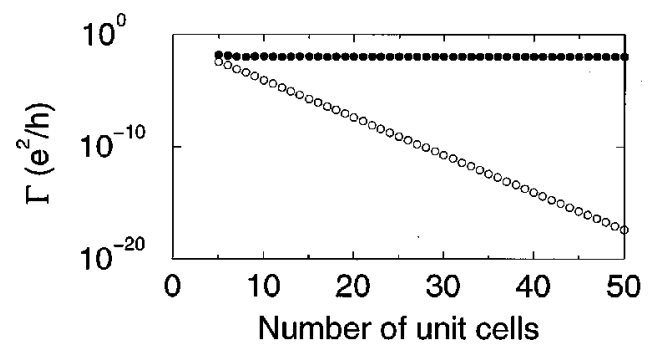

FIG. 2. Total conductances $\Gamma_{\mathrm{FM}}$ (filled circles) and $\Gamma_{\mathrm{AF}}$ (open circles) per unit area as functions of the number of unit cells $n$.

configuration the curves for $T_{\mathrm{AF}}^{\uparrow}\left(E_{\perp}\right)$ and $T_{\mathrm{AF}}^{\downarrow}\left(E_{\perp}\right)$ coincide and are similar to that for $T_{\mathrm{FM}}^{\downarrow}$, exhibiting the same transmission gap and having the first transmission band suppressed by the potential height in one of the magnetic layers. It follows that if the transmission gap contains $E_{F}$, all conductances but $\Gamma_{\mathrm{FM}}^{\uparrow}$ vanish. In other words, $\Gamma_{\mathrm{AF}}=0$ and $\Gamma_{\mathrm{FM}}$ $=\Gamma_{\mathrm{FM}}^{\uparrow}$ so that, in CPP geometry, the system behaves as an insulator in the AF configuration and as a conductor in the FM one, leading to an infinitely large MR ratio. The spacer thickness can always be chosen so that the ferromagnetic layers are coupled antiferromagnetically. In this case, an insulator-to-metal transition can be driven by an externally applied magnetic field that saturates the magnetization, making the system behave as a magnetic logical gate or switch. In addition, in the FM configuration, it acts as a spin filter, which may be useful in performing quantum bit operations required for quantum computing. 7

The appearance of gaps in the transmission curves is directly related to the opening of the spacer Fermi surface (FS) in the modulation direction, i.e., perpendicular to the layers. ${ }^{6}$ Both effects are connected with quantum interference processes associated with the spacer superlattice structure, so that the position, width, and number of gaps can be tuned by varying the potential heights and widths. The opening of the spacer FS has an important consequence since it may lead to both PMR and an exponential decay of the exchange coupling between the ferromagnetic layers as a function of supercell repeats. ${ }^{8}$ Therefore, the magnetic field necessary to change the multilayer configuration from AF to FM can be made as small as necessary, which is highly desirable from the point of view of using these systems as low-field magnetic sensors. It should be noticed that, for small values of $n$, quantum tunneling across the spacer gives rise to finite but small transmission in the gap region, leading to nonvanishing AF conductances and finite MR ratios. Clearly, as $n$ increases $\Gamma_{\mathrm{AF}}$ goes exponentially to zero, whereas $\Gamma_{\mathrm{FM}}$ remains finite, as shown in Fig. 2 for $E_{F}=0.22$ and the other parameters as in Fig. 1. Hence, the MR ratio grows exponentially with $n$ and may reach values much larger than CMR, but for much smaller saturation fields.

Clearly, the occurrence of the PMR regime depends on the right positioning of the transmission gap with respect to the Fermi energy. As already pointed out, this can be accomplished by tuning the spacer supercell size. In fact, Fig. 3 shows the MR ratio calculated as a function of $a$ for $b=a$, $E_{F}=0.22$, and the remaining parameters as in Fig. 1. As $a$ increases, the transmission gap, initially above $E_{F}$, moves downwards and crosses the Fermi energy. Consequently, the 


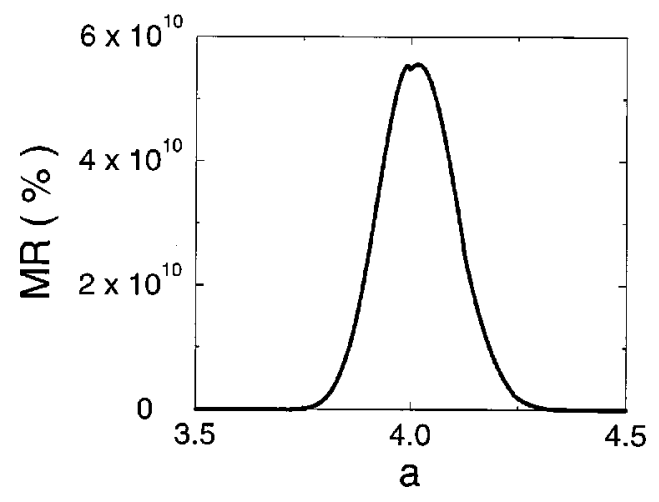

FIG. 3. Magnetoresistance as a function of the thickness $a$.

MR goes from the usual GMR regime to PMR, returning to GMR when the transmission gap is entirely below $E_{F}$. In the PMR regime, the maximum of MR is controlled by the number $n$ of supercell repeats. In the present case $(n=30)$ it reaches $10^{10} \%$, which is several orders of magnitude greater than CMR.

In conclusion, a new spin polarized transport regime can be obtained in magnetic layers separated by periodically modulated spacers. Our results clearly illustrate the possibility of controlling transport and magnetic properties of these systems by conveniently choosing the magnetic materials and adjusting the spacer modulation in order to take advantage of the quantum interference effects within the structure.
The occurrence of an insulating phase eliminates the difficulty usually found in the CPP geometry of measuring exceedingly small resistances. As discussed above, such systems are extremely attractive from the point of view of both basic research and technological applications.

The authors would like to dedicate this letter to Professor David M. Edwards on the occasion of his retirement from teaching. They thank A. Bruno Alfonso for helpful discussions. Financial support from CNPq and FAPERJ of Brazil, the Brazilian Academy of Science, and the Royal Society (U.K.) is gratefully acknowledged.

${ }^{1}$ M. N. Baibich, J. M. Broto, A. Fert, F. Nguyen Van Dau, F. Petroff, P. Etienne, G. Creuzet, A. Friederich, and J. Chazelas, Phys. Rev. Lett. 61, 2472 (1988).

${ }^{2}$ J. Q. Xiao, J. S. Jiang, and C. L. Chien, Phys. Rev. Lett. 68, 3749 (1992); A. E. Berkowitz, J. R. Mitchel, M. J. Carey, A. P. Young, S. Zhang, F. E. Spada, F. T. Parker, A. Hutten, and G. Thomas, ibid. 68, 3745 (1992).

${ }^{3}$ R. Schad, C. D. Potter, P. Belien, G. Verbanck, V. V. Moschchalkov, and Y. Bruynseraede, Appl. Phys. Lett. 64, 3500 (1994).

${ }^{4}$ R. Von Helmolt, J. Wecker, B. Holzapfel, L. Schultz, and K. Samwer, Phys. Rev. Lett. 71, 2331 (1993); S. Jin, T. H. Tiefel, M. Mc Cormack, R. A. Fastnacht, R. Ramesh, and L. H. Chen, Science 264, 413 (1994).

${ }^{5}$ J. Mathon, Phys. Rev. B 54, 55 (1996).

${ }^{6}$ K. M. Schep, P. J. Kelly, and G. E. W. Bauer, Phys. Rev. B 57, 8907 (1998).

${ }^{7}$ D. P. DiVicenzo, Science 270, 255 (1995).

${ }^{8}$ M. S. Ferreira, J. d'Albuquerque e Castro, R. B. Muniz, and L. C. Lopes, Phys. Rev. B 58, 8198 (1998). 